\title{
The Value of Identifying External Branch of Superior Laryngeal Nerve in Post-Operative Voice Performance: A Randomized Clinical Trial
}

\author{
Mehdi Asgari, ${ }^{1}$ Mohammad Hossein Sarmast Shoushtari, ${ }^{1}$ Mojtaba Omidvar,, ${ }^{1}$ Gholam Reza \\ Shamsaie, ${ }^{2}$ Abdolhassan Talaiezadeh, ${ }^{1}$ and Davood Kashipazha ${ }^{2}$ \\ ${ }^{1}$ Department of Surgery, Ahvaz Jundishapur University of Medical Sciences, Ahvaz, Iran \\ ${ }^{2}$ Department of Neurology and Electromyography, Ahvaz Jundishapur University of Medical Sciences, Ahvaz, Iran \\ "Corresponding author: Mojtaba Omidvar, MD, Resident of Surgery, Department of Surgery, Imam Hospital, Azadegan Street, Ahvaz, Iran. Tel: +98-9166511754, E-mail: \\ omidvar_md@yahoo.com
}

Received 2016 January 17; Revised 2016 October 12; Accepted 2016 December 27.

\begin{abstract}
Background: Injury to the external branch of the superior laryngeal nerve (EBSLN) results in deterioration of postoperative voice performance. The aim of this study was to asses the impact of exploring and identifying the EBSLN during thyroidectomy in reducing nerve injury and consequently preserved voice performance.

Methods: Ninety patients, in 2 groups, underwent a thyroidectomy and a total of 122 upper poles were dissected. After 2 to 4 weeks, patients with voice complaints were evaluated by electromyography of cricothyroid muscle.

Results: There were 4 unilateral injuries (10\%) in the explored group whereas in the non-explored group ( 9 unilateral and 2 bilateral) $(\mathrm{P}=0.28)$, there were $11(22 \%)$. A total of $7.4 \%$ of 54 upper thyroid poles injuries were observed in the explored group whereas $16 \%$ injuries of 68 upper thyroid poles in the non-explored group $(\mathrm{P}=0.12)$.

Conclusions: The injury to the EBSLN decreased when upper thyroid pole dissection was accompanied with nerve exploring. The EBSLN should be explored and identified during the thyroidectomy because it makes a noticeable change in the postoperative voice performance.
\end{abstract}

Keywords: Laryngeal Nerves, EBSLN, Electromyography, Thyroidectomy, Voice Quality

\section{Background}

Thyroid surgery is a worldwide procedure that is performed because of different indications. There are several complications after a thyroidectomy and the quality of voice is one of the most important problems. The voice changes after a thyroidectomy is common and in many cases, recurrent laryngeal nerve (RLN) is not injured (1-4). The voice changes after a thyroidectomy are multifactorial, such as injury to the external branch of superior laryngeal nerve (EBSLN) and RLN, endotracheal intubation, laryngotracheal fixation, and strap muscles dysfunction (2).

The EBSLN is the motor supply of the cricothyroid muscle and is the tensor of the vocal cord (5-7). Common symptoms in patients with EBSLN injury include easy voice fatigue, hoarseness and volume changes on loud phonation, difficulties in high pitch sounds and alteration in the fundamental speaking frequency $(2,5-8)$. In order to preserve voice performance and to reduce injury to the EBSLN, there is a different approach in thyroid surgery. Many of surgeons during dissection on the superior thyroid pole try to save the EBSLN by ligation of the superior thyroid pedicle close on superior thyroid pole. Other surgeons may try to explore to identify the nerve, while some believe in the use of intraoperative nerve stimulator to identify the nerve (2, 9).

Several studies have proposed that exploring and identifying the EBSLN during thyroidectomy result in more saved nerve and consequently better post-operative voice performance $(4,6,8)$. Oppositely, there are studies that questioned any benefit for exploring the $\operatorname{EBSLN}(10)$.

In this study we assessed the impact of exploring and identifying of the EBSLN during thyroidectomy in reducing nerve injury and consequently preserved voice performance. There were some objectives for this study, firstly to measure the value of EBSLN explore in preserving the nerve and secondly to suggest a new protocol and algorithm for approach to the voice problem after thyroidectomy.

\section{Methods}

\subsection{Study Design}

This randomized clinical study was performed at 2 general hospitals in Ahvaz, Southwest Iran, from September 2010 to September 2012. Patients have been divided by random permutation into 2 groups. We calculated the sample 
size with an alpha error of $5 \%$ and a beta error of $10 \%$, resulting in a $90 \%$ power.

$n=\frac{\left(z_{1-\frac{\alpha}{2}}+z_{1-\beta}\right)^{2}\left[p_{1}\left(1-p_{1}\right)+p_{2}\left(1-p_{2}\right)\right]}{\left(p_{1-} p_{2}\right)^{2}}$

\subsection{Study Population}

Ninety patients were randomly divided into 2 groups. A total of 40 patients were assigned in the explored group, with exploring to identify the EBSLN and individual ligation of upper pole vessels. In addition, 50 patients were assigned in non-explored group, with ligation of the superior vascular pedicle close on the upper pole, without exploration and/or identification of the EBSLN. Totally 122 upper poles were dissected: 54 in the explored group and 68 in the non-explored group (Table 1). The patients with indication for thyroidectomy were included in the study. Exclusion criteria were prior neck surgery including thyroidectomy and radical neck dissection, and also a history of radiation to neck. This study was approved by the ethics committee of Ahvaz Jundishapur University of Medical Sciences and all the patients were informed and consent forms obtained from them. Ethic registration: ajums.rec.1392.11.

Table 1. Demographic, Characteristics, Diseases and Type of Surgical Procedures

\begin{tabular}{|c|c|c|c|}
\hline Variable & Explored & Non-Explored & PValue \\
\hline Age, $y$ & $39.5 \pm 10.8$ & $39.5 \pm 12.0$ & 0.90 \\
\hline No. Pts & 40 & 50 & $0.13^{\mathrm{a}}$ \\
\hline Female, No.\% & $35(87.5)$ & $40(80)$ & $0.34^{\mathrm{a}}$ \\
\hline Male, No. \% & $5(12.5)$ & $10(20)$ & $0.34^{\mathrm{a}}$ \\
\hline Mean age, $y$ & $39.5 \pm 10.8$ & $39.5 \pm 12$ & $0.90^{\mathrm{b}}$ \\
\hline \multicolumn{4}{|l|}{ Disease } \\
\hline MNG & 17 & 23 & $0.74^{\mathrm{a}}$ \\
\hline $\begin{array}{l}\text { Solitary thyroid nodule } \\
\text { (benign) }\end{array}$ & 19 & 20 & $0.47^{\mathrm{a}}$ \\
\hline Graves & 3 & 4 & $0.93^{\mathrm{a}}$ \\
\hline Cancer & 1 & 3 & $0.42^{\mathrm{a}}$ \\
\hline Thyroid volume on US & $52.4 \pm 23.3$ & $58.6 \pm 24.8$ & $0.21^{\mathrm{b}}$ \\
\hline \multicolumn{4}{|l|}{ Procedure } \\
\hline Total thyroidectomy & 5 & 8 & $0.63^{\mathrm{a}}$ \\
\hline Subtotal & 16 & 22 & $0.70^{\mathrm{a}}$ \\
\hline Lobectomy & 19 & 20 & $0.47^{\mathrm{a}}$ \\
\hline
\end{tabular}

\subsection{Surgical Technique}

An indirect laryngoscopy was performed to assess the vocal cord for the patients before the surgery. A standard thyroidectomy was performed by a senior endocrine surgeon and a senior surgery resident. Accordingly, the thyroid was exposed through a Kocher transverse collar incision, raising sub-platysmal flaps and dividing strap muscles in the midline. After medial rotation and ligation of the middle thyroid vain, identifying the superior thyroid pole began by retracting the thyroid inferiorly and medially. The upper pole was mobilized and elevated using a right angle clamp, or was encircled with an umbilical tape. Then, a gentle traction was made with the tape medially and inferiorly and a second gentle traction to the lateral side of the upper pole by a haemostat clamp, as explained in lateralization technique (8). With this maneuver, upper pole components were easily identified; a careful dissection was carried out in avascular cricothyroid space to identify the EBSLN. The dissection plane should be as close as possible on the upper pole and vessels skeletonized and ligated individually. In this study we used a new algorithm (Figure 1), in order to a systematic approach to different injuries of EBSLN or the RLN. According to this new algorithm, different injuries were assessed based on the different complaints and their severity. After the surgery, during a period of 2 - 4 weeks, all patients were visited. The patients with any voice changes and complaints, including easy voice fatigue, difficulties in high pitch sounds, hoarseness, loudness, and alteration in the fundamental speaking frequency, were evaluated by electromyography (LEMG) of cricothyroid muscle (6). Electromyography of the cricothyroid muscle was performed 3-4 weeks after the thyroidectomy to identify and classify nerve injury. All patients were aware that cricothyroid EMG was a diagnostic procedure and would not help them as a treatment. This was because the cricothyroid EMG was an invasive procedure and might not be well tolerated in many patients.

Patients with suspected injury to the EBSLN were reevaluated after 3 months to identify the voice change due to the temporary and permanent damage. Patients with hoarseness were examined with indirect laryngoscopy, if any abnormality were detected in the vocal cord aperture, video-laryngeal-stroboscopy were performed to role-out RLN injury.

\subsection{Statistical Analysis}

Chi-Square test, t-test and Fisher exact test were the used analytic tests. Results are presented as mean \pm SD and $\mathrm{P}<0.05$ was considered significant with using SPSS18. 
Figure 1. Approach to Voice Complain After Thyroidectomy

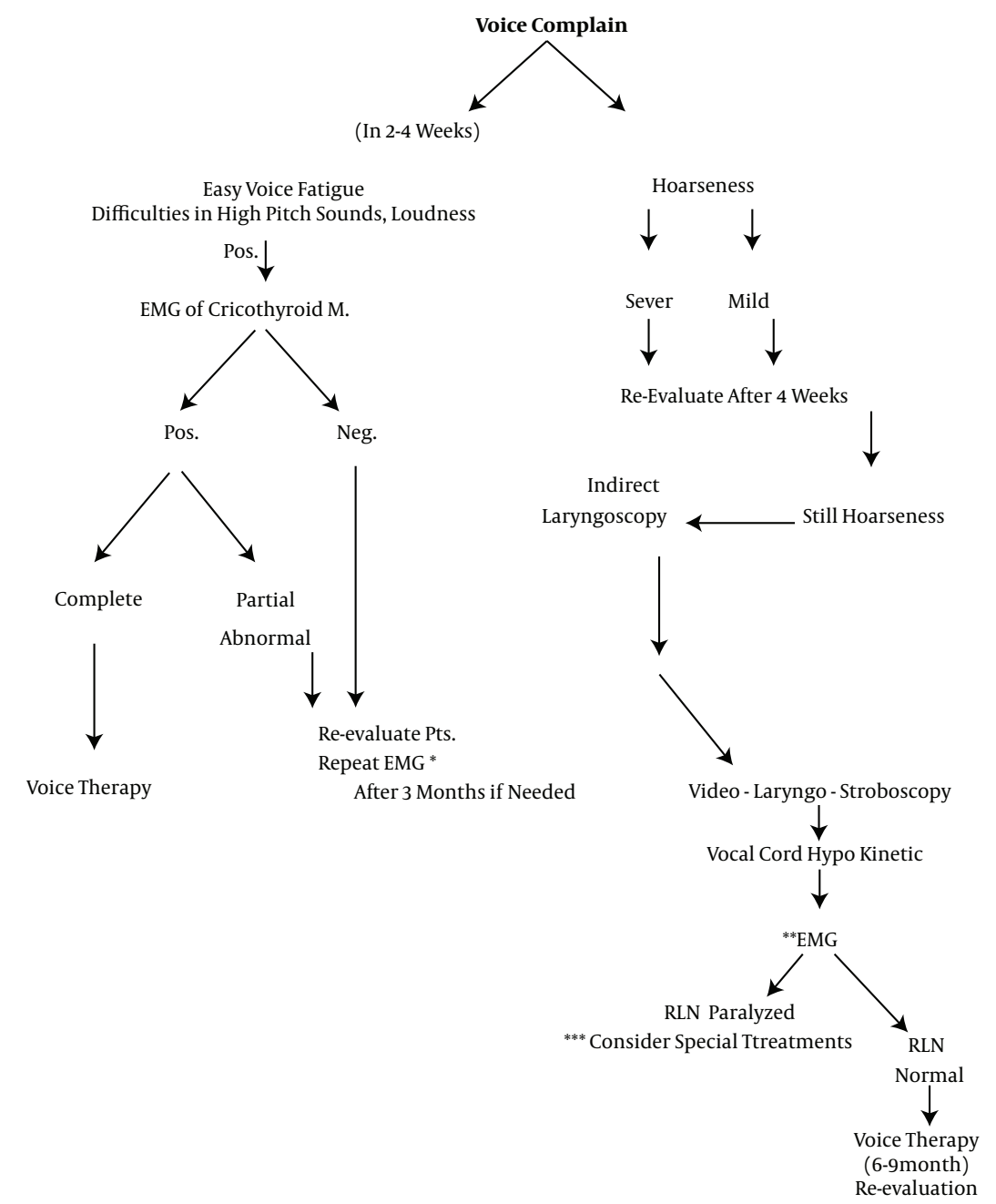

${ }^{*}$ If complaint still presents. ${ }^{* *}$ Intrinsic laryngeal muscle EMG (refer to otolaryngologist). ${ }^{* * *}$ Laryngeal re-innervation, vocal fold injection medialization, (commonly injected agents include hyaluronic acid gels, autologous fat, collagen, micronized human dermis, methylcellulose gel, and calcium hydroxyapatite paste.) (11).

\section{Results}

This study was conducted on the aged and sex match groups (Table 1). The female to male ratio was 7:1 in the explored group and 4:1 in the non-explored group. The mean age in the explored and non-explored groups were $39.5 \pm$ 10.8 and $39.5 \pm 12.0$, respectively $(\mathrm{P}=0.9)$.

In the explored group, there were 4 patients (10\%) with unilateral injuries. In the non-explored group, there were 11 patients (22\%) with injuries ( 9 unilateral and 2 bilateral) $(\mathrm{P}=0.28)$.

In the explored group, there were $7.4 \%$ injuries in 54 upper poles and in the non-explored group, $16 \%$ injuries in 68 upper poles $(\mathrm{P}=0.12)$.
Hoarseness was detected in 9 patients (22.5\%) of the explored group and 13 patients (26\%) of the non-explored $\operatorname{group}(\mathrm{P}=0.7)$.

The nerve was identified in 18 upper poles (33\%), of the explored group, however, it was not seen in the nonexplored group $(\mathrm{P}<0.001)$.

We did not attempt to classify nerves as Barczynski et al.'s classification (9)(Table 2). According to our results and other studies, we proposed an algorithm for approach to voice complaints and complications after surgical procedure on neck especially thyroidectomy (Figure 1). 
Table 2. Results After Thyroidectomy in Explored and Non-Explored Groups

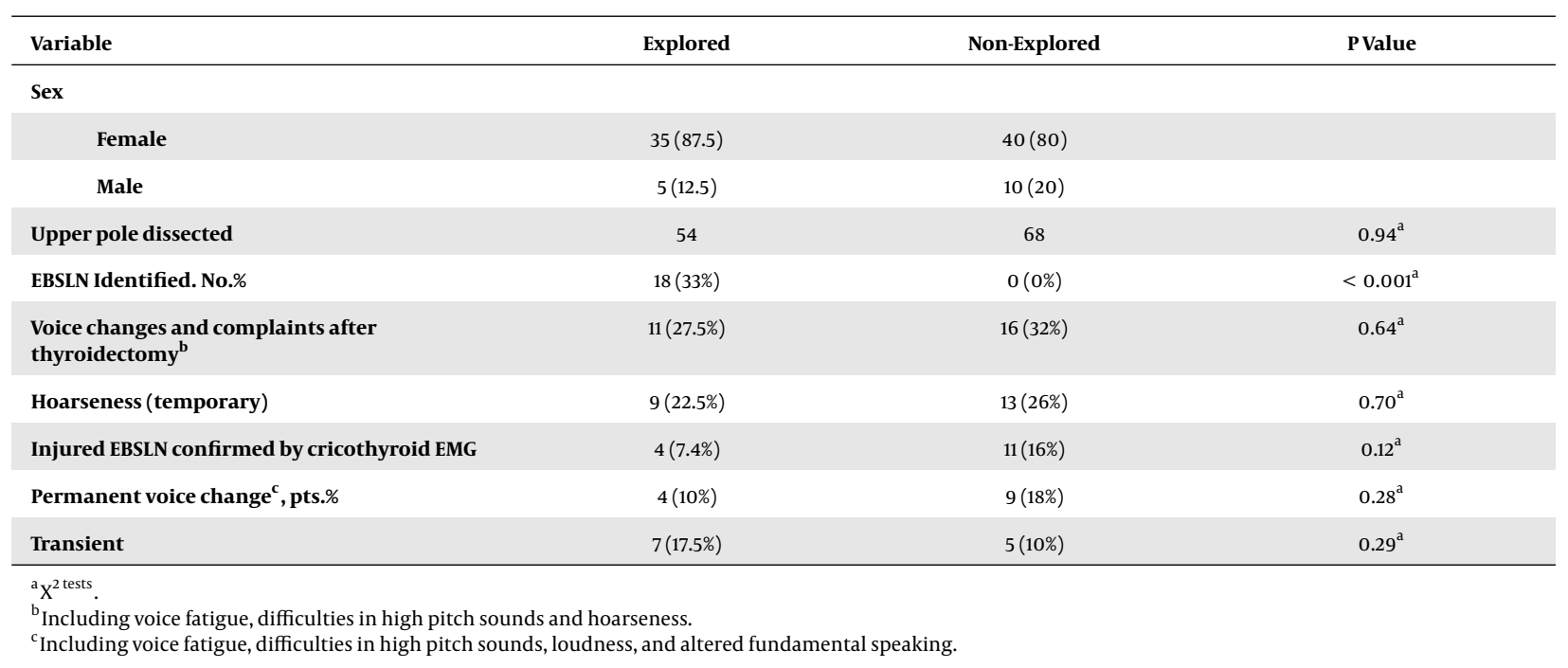

\section{Discussion}

Our study showed that exploration and visual identification of the EBSLN could reduce nerve injury and consequently post-operative voice performance and better outcome. The EBSLN, descends deep to the superior thyroid artery, crosses the potential avascular cricothyroid space and enters cricothyroid muscle along with a cricothyroid artery (5-7). This pathway has made it vulnerable to injury during thyroidectomy (4). The EBSLN supply motor nerve of the cricothyroid muscle and tensor of vocal cords (5-7). Anatomical classification by Cernea et al. (1992) described the relationship between EBSLN and superior thyroid artery (12). According to the Cernea classification, type 2b was considered as high risk for injury and was detected in up to $20 \%$ of individuals. Injury to this nerve leads to voice fatigue during long speeches, impairment in hitting the high notes, loading sounds, difficulties in high pitch sounds, and alteration in the fundamental speaking frequency (5-8). Voice problems are more prominent in women, professional speakers, and especially, singers $(2,7)$.

In this study we had $7.4 \%$ of injuries to the EBSLN in 54 dissected upper poles, (10\% in 40 patients) in the explored group, versus $16 \%$ injuries in 68 dissected upper poles in the non-explored (18\% in 50 patients). As a result, it showed the injury rose nearly two times in the non-explored group as explained, which was considerable. In the present study, the EBSLN was identified by vision in 18 upper poles (33\%), in the explored group. It was relatively low, however, we avoided making an incision on strap muscles to facilitate identification of the nerve because it might deteriorate postoperative voice performance (2). The true incidence of the EBSLN injury after thyroidectomy is not clear, but incidence of the injury according to electromyography of cricothyroid muscle ranges from 0 to $58 \%(7,13)$. The rate of intraoperative nerve identification has been reported 10 to $80 \%(8,13)$. Friedman et al. (14) explained that in more than $85 \%$, the EBSLN could be identified in the junction of the inferior constrictor muscle and cricothyroid muscle. He also mentioned that until the EBSLN has been identified, it is at risk in all patients.

There are studies in support of our conclusion while there are other studies that have opposite conclusion. In support of this study we can note that the study of Hurtado et al.'s study (15), there were 25 nerve injury (50\%) without exploring and 13(26\%) nerve injury with dissection and exploring. This means that the rate of nerve injury decreased in almost half of the patients when exploring the nerve. Other studies that support our view is the study of Mishra et al., (8) they reported no nerve injury after exploring the upper pole. Friedman et al.' study showed that EBSLN should be explored during thyroidectomy and detection is possible in most cases. They concluded that preservation of the EBSLN maintains optimal function of the larynx (14). In opposite of our studies conclusion, Bellatone et al. (10) showed no difference in the rate of injury to the EBSLN during dissecting the upper pole with or without exploration and they questioned needs for exploration of the nerve.

The use of neuromonitoring with nerve stimulator has been evaluated in some studies with controversial results $(2,9)$. Barczynski et al. (9) concluded that the use of intra operative nerve monitoring can considerably improve 
the identification rate of the EBSLN during thyroidectomy. Another study proposed different conclusions; Aina and Hisham (16) reported lack of benefit of a nerve stimulator and neuromonitoring to identify the type II EBSLN.

About 1 in every 10 patients experienced temporary injury to the RLN after surgery, with longer lasting voice problems in up to 1 in 25 (17). Temporary injury to the RLN can be presented with hoarseness. Another reason for hoarseness and voice change is intubation trauma, laryngeal irritation, edema or injury from airway management (17). In the present study temporary hoarseness was observed, $22.5 \%$ in the explored group and $26 \%$ in the nonexplored group.

In EBSLN injury on visualization (laryngoscopy), the edge of the affected vocal cord looks irregular and/or wavy; it usually lies on lower than the other side, it is sometimes shorter than the normal side and flaccid, and bulged during expiration $(17,18)$. Laryngeal electromyography (LEMG) of the cricothyroid muscles have been introduced as the gold standard in the diagnosis of EBSLN paralysis. It is a more reliable test to detect the s denervation rather than visualization technics such as flexible laryngoscopy and stroboscopy $(6,17)$. However, LEMG has limitations (6). Accuracy of LEMG is dependent to the experience of the person who performs and interprets the test. The result can be false if the EMG needle penetrate to the surrounding muscles like straps. A combination of visualisation and symptoms of the patient and LEMG will increase the accuracy of diagnosis (6). In our study we used the LEMG in combination with patients symptoms to increase the reliability of the results.

To better visual control of the EBSLN, the sterno-thyroid muscle must be incised $(9,12)$. Many thyroid surgeons try to prevent injury to this nerve by close ligation on upper thyroid pole. Even with the close ligation on the superior thyroid pedicle, there is at least $20 \%$ risk of injury to the EBSLN, when not identified, (because of the at risk type (2b) as Cornea's classification (12). The present study had some advantages. We used a new protocol for approach to the voice complains after thyroidectomy (Figure 1). We proposed this protocol because it could be used as a reliable approach to voice complains after thyroidectomy.

\subsection{Suggestion}

It must be considered that even with exploring and identifying the EBSLN during the thyroidectomy, there is always risk for injury to this nerve because of its anatomical position and characteristics. We do not recommend too much persist on identifying the nerve because it could lead to more injury to the EBSLN, so this should be avoided.

\subsection{Conclusion}

This study showed that exploration of EBSLN during thyroidectomy decreased the rate of injury and had a noticeable effect on voice performance of the patients.

\section{Acknowledgments}

This study was a postgraduate thesis of Dr. M. Omidvar and financial support provided by Ahvaz Jundishapur University of Medical Sciences from Ahvaz, Iran. The authors would like to thank Ahvaz Jundishapur University of Medical Sciences for their financial support of this approved research project. Our special thanks goes to Dr. Nader Paziar, the head of the Golestan Hospital Clinical Development Research Unit for his great helps and contributions in this study. We would also like to thank Molook Salemzadeh, the member of Golestan Hospital Clinical Development Research Unit for her contributions.

\section{Footnotes}

Authors' Contribution: All authors contributed equally in planning and carrying out this project.

Financial Disclosure: We had no financial interests related to the material in the manuscript.

Funding/Support: Financial support of this study was provided by Ahvaz Jundishapur University of Medical Sciences. There were no other Sponsors.

\section{References}

1. Sinagra DL, Montesinos MR, Tacchi VA, Moreno JC, Falco JE, Mezzadri NA, et al. Voice changes after thyroidectomy without recurrent laryngeal nerve injury. J Am Coll Surg. 2004;199(4):556-60. doi: 10.1016/j.jamcollsurg.2004.06.020. [PubMed:15454138].

2. Barczynski M, Konturek A, Stopa M, Honowska A, Nowak W. Randomized controlled trial of visualization versus neuromonitoring of the external branch of the superior laryngeal nerve during thyroidectomy. World J Surg. 2012;36(6):1340-7. doi: 10.1007/s00268-012-1547-7. [PubMed: 22402975].

3. Lombardi CP, Raffaelli M, D'Alatri L, Marchese MR, Rigante M, Paludetti G, et al. Voice and swallowing changes after thyroidectomy in patients without inferior laryngeal nerve injuries. Surgery. 2006;140(6):1026-32. doi: 10.1016/j.surg.2006.08.008. [PubMed: 17188153] discussion 1032-4.

4. Pagedar NA, Freeman JL. Identification of the external branch of the superior laryngeal nerve during thyroidectomy. Arch Otolaryngol Head Neck Surg. 2009;135(4):360-2. doi: 10.1001/archoto.2009.9. [PubMed: 19380357].

5. Arnold GE. Physiology and pathology of the cricothyroid muscle. Laryngoscope. 1961;71:687-753. doi: 10.1288/00005537-19610700000002. [PubMed: 13684376 ].

6. Orestes MI, Chhetri DK. Superior laryngeal nerve injury: effects, clinical findings, prognosis, and management options. Curr Opin Otolaryngol Head Neck Surg. 2014;22(6):439-43. doi: 10.1097/MOO.0000000000000097. [PubMed: 25136863]. 
7. Marchese-Ragona R, Restivo DA, Mylonakis I, Ottaviano G, Martini A, Sataloff RT, et al. The superior laryngeal nerve injury of a famous soprano, Amelita Galli-Curci. Acta Otorhinolaryngol Ital. 2013;33(1):67-71. [PubMed: 23620644].

8. Mishra AK, Temadari H, Singh N, Mishra SK, Agarwal A. The external laryngeal nerve in thyroid surgery: the 'no more neglected' nerve. Indian J Med Sci. 2007;61(1):3-8. [PubMed:17197732].

9. Barczynski M, Randolph GW, Cernea CR, Dralle H, Dionigi G, Alesina $\mathrm{PF}$, et al. External branch of the superior laryngeal nerve monitoring during thyroid and parathyroid surgery: International Neural Monitoring Study Group standards guideline statement. Laryngoscope. 2013;123 Suppl 4:S1-14. doi: 10.1002/lary.24301. [PubMed: 23832799].

10. Bellantone R, Boscherini M, Lombardi CP, Bossola M, Rubino F, De Crea $\mathrm{C}$, et al. Is the identification of the external branch of the superior laryngeal nerve mandatory in thyroid operation? Results of a prospective randomized study. Surgery. 2001;130(6):1055-9. doi: 10.1067/msy.2001.118375. [PubMed: 11742338].

11. Mallur PS, Rosen CA. Vocal fold injection: review of indications, techniques, and materials for augmentation. Clin Exp Otorhinolaryngol. 2010;3(4):177-82. doi: 10.3342/ceo.2010.3.4.177. [PubMed: 21217957].

12. Cernea CR, Ferraz AR, Nishio S, Dutra AJ, Hojaij FC, dos Santos LR. Surgical anatomy of the external branch of the superior laryngeal nerve.
Head Neck. 1992;14(5):380-3. [PubMed: 1399571].

13. Jansson S, Tisell LE, Hagne I, Sanner E, Stenborg R, Svensson P. Par tial superior laryngeal nerve (SLN) lesions before and after thyroid surgery. World J Surg. 1988;12(4):522-7. [PubMed: 3420935].

14. Friedman M, LoSavio $P$, Ibrahim $H$. Superior laryngeal nerve identification and preservation in thyroidectomy. Arch Otolaryngol Head Neck Surg. 2002;128(3):296-303. [PubMed: 11886347].

15. Hurtado-Lopez LM, Pacheco-Alvarez MI, Montes-Castillo Mde L, Zaldivar-Ramirez FR. Importance of the intraoperative identification of the external branch of the superior laryngeal nerve during thyroidectomy: electromyographic evaluation. Thyroid. 2005;15(5):449-54. doi: 10.1089/thy.2005.15.449. [PubMed: 15929666].

16. Aina EN, Hisham AN. External laryngeal nerve in thyroid surgery: is the nerve stimulator necessary?. Eur J Surg. 2001;167(9):662-5. doi: 10.1080/11024150152619282. [PubMed: 11759734].

17. Sulica L. The superior laryngeal nerve: function and dysfunction Otolaryngol Clin North Am. 2004;37(1):183-201. doi: 10.1016/S00306665(03)00175-0. [PubMed: 15062693].

18. Aluffi P, Policarpo M, Cherovac C, Olina M, Dosdegani R, Pia F. Postthyroidectomy superior laryngeal nerve injury. Eur Arch Otorhinolaryngol. 2001;258(9):451-4. [PubMed: 11769989]. 